\title{
A critical evaluation of the interpretation of electrocatalytic nano-impacts
}

Loretta S.Y. Ly, Christopher Batchelor-McAuley, Kristina Tschulik, Enno Kätelhön and Richard

G. Compton*

Department of Chemistry, Physical and Theoretical Chemistry Laboratory, University of Oxford, South Parks Road, Oxford OX1 3QZ, United Kingdom

\section{KEYWORDS}

Gold nanoparticles, proton reduction, electrocatalysis, carbon microelectrode, mass-transport

For submission to: J. Phys. Chem. C 


\begin{abstract}
The kinetics of the proton reduction reaction is studied on a variety of gold surfaces including both macro $\left(\mathrm{r}_{0}=1.0 \mathrm{~mm}\right)$, micro $\left(\mathrm{r}_{0}=4.6 \mu \mathrm{m}\right)$ electrodes and gold nanoparticles $\left(\mathrm{r}_{\mathrm{NP}}=\sim 10 \mathrm{~nm}\right)$ $\mathrm{nm})$. For the gold nanoparticles two complementary methodologies of study are used. First the particles are investigated as part of an ensemble response in an array $\left(\mathrm{k}_{0} \sim 7 \times 10^{-8} \mathrm{~m} \mathrm{~s}^{-1}\right)$. Second, the rate is recorded stochastically at individually impacting nanoparticles $\left(\mathrm{k}_{0} \sim 2 \times 10^{-9} \mathrm{~m} \mathrm{~s}^{-1}\right)$. This apparent decrease in rates on transitioning from the ensemble to individual nanoparticles is understood in terms of the differing connectivity of the nanoparticles to the electrode surface. During the course of the individual catalytic impacts, or 'pulses', the recorded current is found to be highly variable; this variability is taken to originate from the nanoscopic motion of the particle above the electrode interface.
\end{abstract}




\section{INTRODUCTION}

Under optimal conditions the use of electrocatalytic amplification can enable the non-destructive and stochastic detection of single nanoparticles impacting upon a potentiostated electrode. ${ }^{1-3} \mathrm{In}$ these experiments nanoparticles, suspended in solution, are randomly transferred to the surface of an electrode held under potentiostatic control by virtue of their Brownian motion. For the duration of the 'nano-impact', the nanoparticle can mediate electron transfer to/from solution phase molecular species. As well as being a method for the analytical detection and quantification of nanoparticles, ${ }^{4}$ the electrocatalytic impact methodology also holds great promise as a route by which a number of nanochemistry problems may be experimentally probed. One example application is the measurement of individual particle activities via the study of the magnitude of the catalytic current. ${ }^{1}$ Such work will possibly lead to greater insights into the specific structure-activity relationship ${ }^{5}$ of various nanoparticle systems. Additionally, due to the high mass-transport rates to and from the nanoparticle surface - measurement of the electrocatalytic current possibly provides an excellent framework for the investigation of the fundamentals and dynamics of electrochemical processes. Such experiments may help to progress the investigation and validation of the Marcus-Hush formalism, ${ }^{6}$ or highlight coupling between the diffusion and double layers. ${ }^{7}$

Apart from measurement of the magnitude of the catalytic current, the frequency and duration of the nano-impact events also hold information regarding both the mass-transport of a nanoparticle and its possible interaction with the electrified interface. The mass-transport of a particle adjacent to a surface has been predicted to be hindered. ${ }^{8}$ When located at the interface the particle diffusion coefficient perpendicular to the surface asymptotically drops to zero. Movement parallel to the interface is reduced but to a smaller extent. Experimentally, these theoretical predictions have been at least partially verified through the use of optical nanoparticle tracking analysis, ${ }^{9,10}$ however exact resolution of the particle's position and diffusion coefficient is challenging: The electrocatalytic nanoparticle impact experiments may provide another route by which these phenomena can be probed.

Success of the electrocatalytic impact approach is primarily predicated upon the selection of a suitable redox couple for which the electrochemical rate differs dramatically between the 
electrode substrate and the nanoparticle of study. This requirement of a relatively large difference in rates reflects the often significant difference (by orders of magnitude) between the dimensions of the electrode substrate and impacting nanoparticle. ${ }^{\text {a }}$ The use of a surface sensitive or so-called 'inner-sphere" ${ }^{11}$ interfacial redox reaction provides one route by which a large enough difference in rates may be attained. The hydrogen evolution reaction (HER) is one such example which is simultaneously of academic and industrial interest. ${ }^{12-14}$ Previous work has demonstrated that through the use of a highly-activated carbon fiber microelectrode, ${ }^{3}$ diffusion limited proton reduction can be observed at an individual impacting nanoparticle. For the purposes of analytical nanoparticle detection the use of such active electrodes is not problematic, assuming the background current is relatively constant; however, for detailed studies of individual impact events and their corresponding current traces, low background currents are of paramount importance. Moreover, for the unequivocal resolution of an impacting particle event it is desirable to use low nanoparticle concentrations. The application of dilute nanoparticle solutions has the advantage of helping to ensure diffusional isolation of the nanoparticle.

The work presented herein investigates the HER at both 'individual' and 'arrays of' gold nanoparticles $\left(\mathrm{r}_{\mathrm{NP}} \sim 10 \mathrm{~nm}\right)$. For the impact experiments the mediated reduction reaction is studied at a non-activated carbon fiber electrode using low nanoparticle concentrations (2 pM). These experimental conditions enable the unambiguous measurement of individual nanoparticle impact reactions. Importantly, the rate of electron transfer recorded from the individual particle impacts differs from that measured via the use of a nanoparticle array (i.e. the ensemble average). This difference in measured rates between the two methodologies may either reflect the influence of the differing mass-transport regimes of a particle in the two environments (array versus isolated), or alternatively the decreased kinetics may reflect the altered connectivity between the nanoparticle and electrode substrate. In order to illuminate this problem three major experimental observations are made. First, the residence times of the impacting nanoparticles is

a For an interfacial electrochemical redox process the 'irreversibility' of a redox reaction depends not only upon the standard electrochemical rate constant $\left(\mathrm{k}_{0}\right)$ but also varies as a function of the rate of mass-transport to and from the interface. For a fixed rate constant the voltammettric wave appears at greater overpotentials as the electrode size is reduced. 
significant $(>0.5 \mathrm{~s}$ ) implying a strong interaction of the nanoparticle with the electrode surface (adsorption or hindered mass-transport). Second, the catalytic current transients corresponding to the nanoparticle impacts are highly varied in shape. Third, the magnitude of the electrochemical noise increases significantly during the course of a nanoparticle impact. The above observations experimentally evidence the motion of the nanoparticle on the electrochemical interface. The sub-diffusion limited current implies a contact resistance between the electrode and the

nanoparticle. In analogy to in situ scanning tunneling microscopy (STM) ${ }^{15,16}$ this variable current can be understood in terms of reflecting the distance dependence of the induced nanoparticulate catalytic current. Through the study of the current associated with individual nano-impacts this work aims to give physical insight into the processes which control the magnitude of the nanoparticle mediated current. Such that, this work serves to highlight that a complete interpretation of nanoparticle impact experiments will require a full understanding of the masstransport of the nano-material at the electrochemical interface.

\section{EXPERIMENTAL}

Citrate-capped AuNPs, provided by Mintek (Randburg, South Africa), were confirmed to have mean radii of $9.8 \pm 6 \mathrm{~nm}$ via SEM (LEO Gemini II 1530, Zeiss, Oberkochen, Germany). Further information on nanoparticle characterization can be found in the SI section 1. The chemicals used were: perchloric acid (70\%, Sigma-Aldrich) and sodium perchlorate monohydrate (98\%, Sigma-Aldrich). All solutions were prepared using ultrapure water of resistivity $\geq 18.2 \mathrm{M} \Omega \mathrm{cm}$ at $298 \mathrm{~K}$ (Millipore) and were thoroughly degassed with nitrogen prior to all experiments.

\section{Impact experiments}

Impact experiments were conducted at $298 \mathrm{~K}$ using a $\mu$ Autolab III (Metrohm Autolab B.V., Netherlands) and a three electrode set up: a $4.6 \mu \mathrm{m}$ (radius) carbon microelectrode was used as a working electrode, a carbon graphite rod was used as a counter electrode and applied potentials were measured against a saturated mercury/mercury sulphate reference electrode (MSRE, ALS Co., Ltd, Japan, potential vs NHE $=682 \mathrm{mV}$ at $25^{\circ} \mathrm{C}$ ). Prior to each scan, the electrode was polished with $3.0 \mu \mathrm{m}, 1.0 \mu \mathrm{m}$ and $0.1 \mu \mathrm{m}$ diamond spray (Kemet Ltd., UK). 


\section{Cyclic Voltammetry}

A similar three electrode set up was used for cyclic voltammetry experiments but with either a platinum $\left(\mathrm{r}_{0}=0.85 \mathrm{~mm}\right)$, a gold $\left(\mathrm{r}_{0}=1.0 \mathrm{~mm}\right)$ or a gold nanoparticle modified glassy carbon macroelectrode $\left(\mathrm{r}_{0}=1.5 \mathrm{~mm}\right)$ or a gold microelectrode $\left(\mathrm{r}_{0}=4.6 \mu \mathrm{m}\right)$ as a working electrode (both electrodes comprise of 4N [99.99\% purity] gold). The electrodes were polished with alumina ( $3 \mu \mathrm{m}, 1 \mu \mathrm{m}$ and $0.05 \mu \mathrm{m}$ ) for 3 minutes each and then sonicated for 2 minutes before each experiment.

For AuNP modified electrode experiments, a fully polished glassy carbon macroelectrode was dropcast with $3 \mu \mathrm{L}$ of $500 \mathrm{pM}$ AuNP stock solution. The droplet was dried under a stream of nitrogen.

\section{RESULTS AND DISCUSSION}

Initially, in the first section, a summary of the kinetics of $H E R$ at both macro and microelectrodes is presented. Further experimental details on these experiments and results can be found in the SI section 2. This work continues by studying the rate of proton reduction at a gold nanoparticle array (supported on a glassy carbon substrate). Section two evidences the nanoparticle mediation of the proton reduction reaction at individual gold nanoparticles impacting a carbon fibre electrode potentiostated to $-1.9 \mathrm{~V}$ (vs MSRE). Initial analysis of the current transients focuses on their general features such as magnitude and duration. This work is continued in section three which serves to study the gold nano-impacts as a function of the electrode potential. The potential dependence of the magnitude of the nanoparticle mediated current allows the apparent kinetics of the proton reduction at the individual nanoparticles to be measured. The rate of reduction on the individual nanoparticles is found to be significantly suppressed from that recorded at the nanoparticle array. The possible origin of this discrepancy in the rates is discussed. Finally the fourth section studies the Faradaic noise associated the mediated electrochemical process at individual nanoaprticles. Evidence is provided that the recorded noise is too large to be solely associated with instantaneous fluctuations (shot-noise) in the proton concentration local to the nanoparticles. Consequently it is proposed that this increased noise reflects the motion adjacent to the electorchemical interface. 


\section{Proton reduction at gold electrodes and arrays}

Experiments were performed in an aqueous solution containing $0.60 \mathrm{M} \mathrm{NaClO}_{4}$ and $10.0 \mathrm{mM}$ $\mathrm{HClO}_{4}$. From the reductive steady-state current at a gold microdisc electrode $\left(\mathrm{r}_{0}=4.6 \mu \mathrm{m}, \mathrm{i}_{\mathrm{ss}}=\right.$ $157 \mathrm{nA}$ ) the proton diffusion coeffiecient $\left(\mathrm{D}_{\mathrm{H}+}\right)$ was determined to be $8.8 \times 10^{-9} \mathrm{~m}^{2} \mathrm{~s}^{-1}$. Measurement of the formal potential for the proton/hydrogen redox couple was enabled through the use of a platinum macroelectrode $\left(\mathrm{r}_{0}=0.85 \mathrm{~mm}\right)$. The voltammetric mid-point potential for the hydrogen evolution reaction was found to be $-0.799 \mathrm{~V}$ (vs MSRE). This value was taken to accurately reflect the formal potential of the redox couple $\left(E_{f\left(H^{+} / H_{2}\right)}^{\theta}\right)$ and is in good agreement to values found within the literature. ${ }^{17,18}$ In contrast to platinum, proton reduction was found to be fully electrochemically irreversible on gold. At a scan rate of $200 \mathrm{mVs}^{-1}$, on a gold electrode $\left(\mathrm{r}_{0}=1.0 \mathrm{~mm}\right)$ a well-defined reduction wave was observed with a peak at $-1.16 \mathrm{~V}$ (vs MSRE). Tafel analysis yielded a transfer coefficient $\left(\alpha_{\text {Macro }}\right.$ ) of $0.63 \pm 0.03$. Through the use of the commercially available digital simulation software DigiSim ${ }^{\circledR}$ (version 3.0, BASi Technicol, West Lafayette, USA) the electrochemical reaction was modelled as a simple one-electron reduction. In this way the standard electrochemical rate constant $\left(\mathrm{k}_{0, \text { Macro }}\right)$ was measured to be $7.1 \pm 0.3 \times 10^{-8} \mathrm{~m} \mathrm{~s}^{-1}$. Similarly for the gold microelectrode, Tafel analysis yielded a transfer coefficient $\left(\alpha_{\text {Micro }}\right)$ of $0.50 \pm 0.03$. Through the use of an in house developed simulation program ${ }^{4}$ the standard electrochemical rate constant $\left(\mathrm{k}_{0, \text { Micro }}\right)$ was found to be $5.3 \pm 2.0 \times 10^{-7} \mathrm{~m} \mathrm{~s}^{-1}$. Hamelin et al. found that the rate of hydrogen evolution differed by more than a factor of two when $5 \mathrm{~N}$ versus $4 \mathrm{~N}$ gold was used (the slower rate was found for the purer $5 \mathrm{~N}$ material). ${ }^{19}$ Consequently, this difference in measured kinetics between the macro and microelectrode is significant and most likely reflects the high sensitivity of the reaction towards impurities in/on the gold metal. ${ }^{14,19,20}$ For the present work this explanation of impurity sensitivity, is corroborated by the greater error associated with the measurement of the standard electrochemical rate constant $\left(\mathrm{k}_{0, \text { Micro }}\right)$ at the microelectrode, reflecting the more variable surface impurity levels between electrode preparations. 


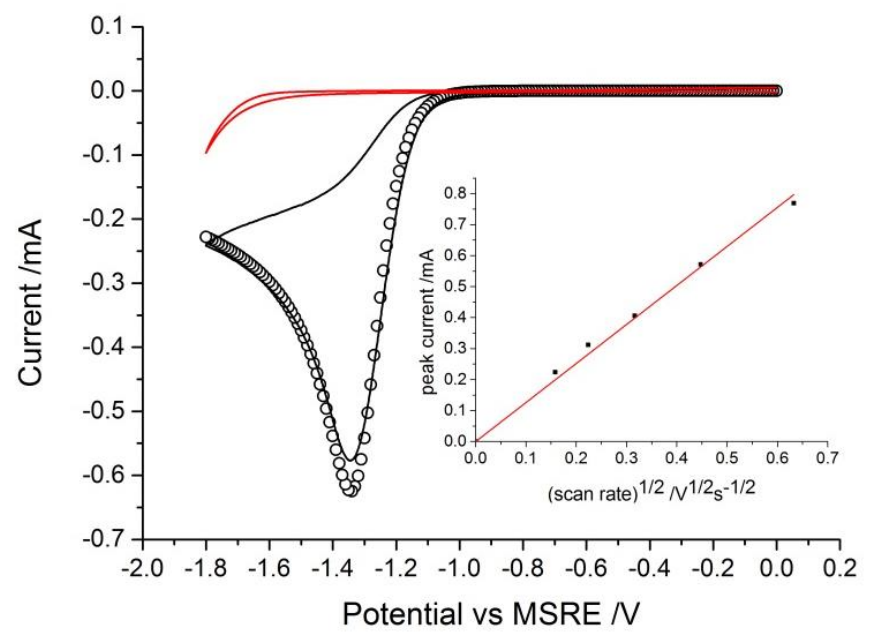

Figure 1 The voltammetric response $\left(200 \mathrm{mV} \mathrm{s}^{-1}\right)$ of a bare (-) and gold nanoparticle modified (-) glassy carbon electrode in $0.6 \mathrm{M} \mathrm{NaClO}_{4}$ and $10 \mathrm{mM} \mathrm{HClO}_{4}$. Also shown (circles) is the simulated voltammetric response for the nanoparticle array (3.9\% surface coverage) with a standard electrochemical rate constant $\left(\mathrm{k}_{0}\right)$ of $6.6 \times 10^{-8} \mathrm{~m} \mathrm{~s}^{-1}, E_{f}^{\theta}=-$ 0.799V (vs MSRE), $r_{\text {geo }}=1.5 \times 10^{-3} \mathrm{~m}$.

Having quantified the hydrogen evolution reaction at 'bulk' gold, the work shall now study the gold nanoparticle $\left(\mathrm{r}_{\mathrm{NP}} \sim 10 \mathrm{~nm}\right)$ ensemble response towards proton reduction. A glassy carbon electrode was modified via drop-casting $3.0 \mu \mathrm{L}$ of the gold nanoparticle stock solution on to the electrode surface. This quantity of nanoparticles yields a surface coverage of $3.9 \%$ (projected electrode area). The voltammetric response of the gold nanoparticle array towards proton reduction was studied in the aqueous electrolyte containing $0.6 \mathrm{M} \mathrm{NaClO}_{4}$ and $10 \mathrm{mM} \mathrm{HClO}_{4}$, a representative voltammogram is depicted in Figure 1.

First, in the absence of nanoparticles the voltammetric response of a bare glassy carbon electrode did not exhibit any significant proton reduction within the electrochemical window of study. Second, for the catalyzed proton reduction peak, in the presence of the gold nanoparticles, the peak current was found to vary linearly with the square-root of scan rate (inlay Figure 1). This linear variation of the peak height confirms that for the 3.9\% nanoparticle surface coverage there is strong diffusional overlap between the adjacent nanoparticles in the array, consequently, the diffusion profile at the electrode will be linear. ${ }^{21}$ Third, Tafel analysis of the voltammetric response yielded a transfer coefficient $\left(\alpha_{\mathrm{NP}}\right.$,ensemble $)$ of $0.47 \pm 0.02$ (SI section 3 ). The theory of heterogeneous electrodes has been previously treated theoretically by Amatore et al. ${ }^{22}$ More recently, this work has been extended to allow consideration of the response of a nanoparticle 
array. ${ }^{23}$ Using this theory the voltammetric response of the gold nanoparticle modified electrode was simulated and a value for the standard electrochemical rate constant $\mathrm{k}_{0, \mathrm{NP} \text {,ensemble was found }}$ to be $6.6 \pm 2.1 \times 10^{-8} \mathrm{~m} \mathrm{~s}^{-1}$. Figure 1 also depicts an example of the simulated voltammetric wave (circles) for the nanoparticle array. Table 1 summarises the measured kinetic data for the rate of proton reduction on the various gold electrodes.

Table 1: Comparative proton reduction kinetics

Electrode Transfer Coefficient $(\alpha) \quad$ Standard Electrochemical rate constant $\left(\mathrm{k}_{0}\right)$

$\begin{array}{ccc}\text { Au macro }\left(\mathrm{r}_{0}=1.0 \mathrm{~mm}\right) & 0.63 \pm 0.03 & 7.1 \pm 0.3 \times 10^{-8} \mathrm{~m} \mathrm{~s}^{-1} \\ \text { Au micro }\left(\mathrm{r}_{0}=4.6 \mu \mathrm{m}\right) & 0.50 \pm 0.03 & 5.3 \pm 2.0 \times 10^{-7} \mathrm{~m} \mathrm{~s}^{-1} \\ \text { Glassy carbon/Au NP Array } & 0.47 \pm 0.02 & 6.6 \pm 2.1 \times 10^{-8} \mathrm{~m} \mathrm{~s}^{-1} \\ 3.8 \% \text { surface coverage } & & \end{array}$

The difference in the kinetics as recorded for the nano-array versus the 'bulk' gold is worth discussion. For the simulation model used it has been assumed that no agglomeration has occurred for the nanoparticles during the drop-casting process. Consequently, the rate presented for the reduction should be viewed as a lower limit for the proton reduction rate at the gold nanoparticle surface. Even considering the possible limitations of the model the rates for the reduction at the pure gold electrodes and the gold nanoparticle array still show a significant difference. This difference in rates most likely reflects the presence of adsorbed species present on the nanoparticle surface. Capping agents (ligands) play a fundamental role during the chemical synthesis of the gold nanoparticles and also serve to ensure their longer term stability within solution. ${ }^{24}$ For electrochemical experiments these capping agents are often removed prior to experimentation to ensure the 'full' activity of the nanoparticle. ${ }^{25,26}$ However, for the nanoparticle impact experiment such pre-treatment procedures are not easily applicable. Consequently, it is this value for the standard electron transfer rate of $6.6 \pm 2.1 \times 10^{-8} \mathrm{~m} \mathrm{~s}^{-1}$ - as 
measured at the unaltered nanoparticles - that is most pertinent for comparison with the kinetics of the HER at individual and isolated nanoparticles.

\section{Proton reduction at individual gold nanoparticles at $\mathbf{- 1 . 9 V}$ (vs MSRE)}

Having quantified the mean kinetics for the hydrogen evolution reaction at the gold nanoparticle array, the article next focuses on the determination of the reaction rate at individual nanoparticles. This is achieved through the use of the 'nanoimpact' methodology. Here, the nanoparticles are suspended within the electrolyte and due to Brownian motion they sporadically collide with a potentiostated working electrode. Upon collision a potential is induced on the nanoparticle and assuming sufficient driving force a current will be measured corresponding to the redox reaction at the individual nanoparticle. For the present experimental case a carbon fibre microelectrode $\left(\mathrm{r}_{0}=4.6 \mu \mathrm{m}\right)$ was immersed into the aqueous electrolyte $\left(0.60 \mathrm{M} \mathrm{NaClO}_{4}, 10.0\right.$

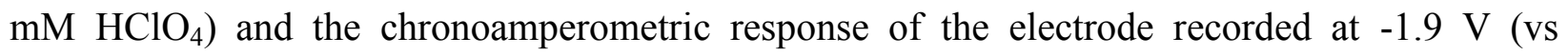
MSRE) both in the presence ( $2 \mathrm{pM})$ and absence of the gold nanoparticles. Note, for the current experimental setup this value of $-1.9 \mathrm{~V}$ (Vs MSRE) represents the most reductive potential that can be potentiostatically applied in the absence of any significant proton reduction occurring directly at the carbon electrode. Figure 2 depicts three representative and contrasting chronoamperometric current transients, in the presence (coloured) and absence (black) of the gold nanoparticles.
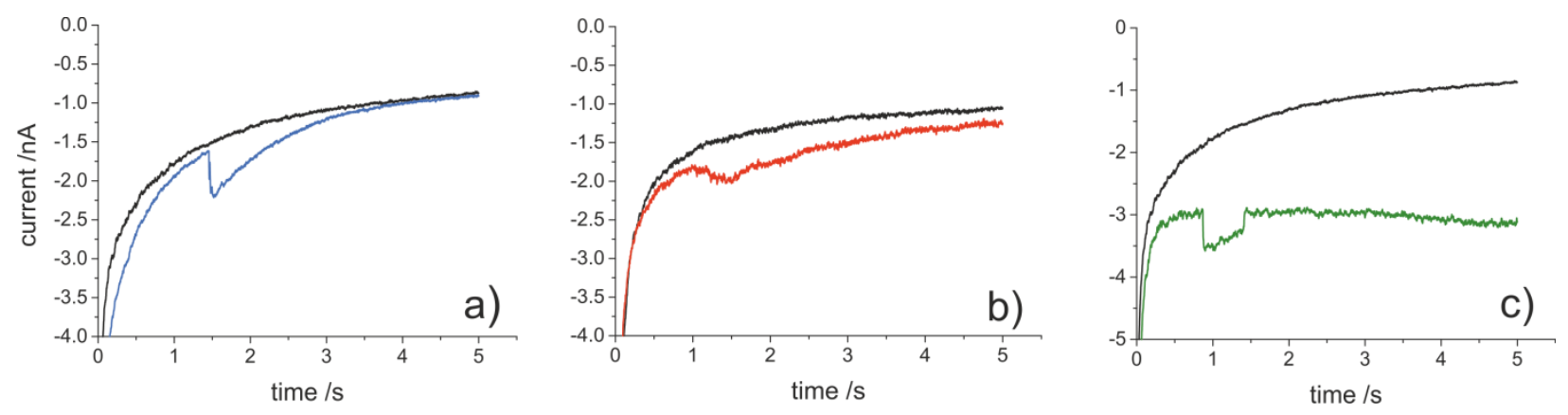

Figure 2: Representative chronoamperometic responses for a carbon micro-electrode $\left(r_{0}=4.6 \mu \mathrm{m}\right)$ potentiostated at $-1.9 \mathrm{~V}$ (vs MSRE) in $0.6 \mathrm{M} \mathrm{NaClO}_{4}$ and $10 \mathrm{mM} \mathrm{HClO}_{4}$, in the presence (red) and absence (black) of 2 pM gold nanoparticles ( $\mathrm{r}_{\mathrm{NP}}$ $=9.8 \pm 6.0 \mathrm{~nm}$ ). All three chronoamperograms exhibit a single current pulse which have been categorized on the basis of their shape a) step on, gradual off (blue), b) gradual on, gradual off (red) and c) step on, step off (green). 
In the presence of the gold nanoparticles the current transient is found to exhibit distinct reductive features that are attributed to proton reduction reaction occurring at individual gold nanoparticles. In order to confirm that these observed current pulses correspond to proton reduction the nanoparticle impact experiment was undertaken at different acid concentrations (5$20 \mathrm{mM} \mathrm{HClO}_{4}$ ). The SI section 4 depicts the experimentally recorded linear correlation between the average pulse height and the solution phase proton concentration. This observation strongly affirms that current traces correspond to the reduction of protons at the transiently impacting gold nanoparticles.

From condsideration of Figure 2, it can be seen that for the recorded pulses the 'shape' of the current traces can vary dramatically. The most rapid current changes occur over $\sim 3.5 \mathrm{~ms}$ i.e. at the bandwidth limit of the current amplifier. These different current traces allow the impact responses to be roughly categorized, where the change in current is either referred to as a 'step' or a 'gradual' transition. Specifically, any change in the pulse height which occurs over a time shorter than $10 \mathrm{~ms}$ is referred to as a 'step' in the current. Consequently, the nanoparticle pulses have been catagorised into four groups depending on their structure. These groups are: 1) 'step on, gradual off' [Figure 2 a)], 2) 'gradual on, gradual off' [Figure 2 b)], 3) 'step on, step off' [Figure 2 c)] and 4) 'gradual on, step off'.

Beyond, the shape of the current traces their duration may also be determined. Figure 3 a) provides a plot of the recorded durations and pulse heights for the electrocatalytic impacts at -1.9 $\mathrm{V}$ (vs MSRE) with $10 \mathrm{mM} \mathrm{HClO}_{4}$ (the pulses have also been classified as in terms of their shape in accordance with the above categories). Due to the chronoamperometry being recorded for a total of 5 seconds, some of the pulses events continue beyond the end of the experimental time frame. Consequently, for these features although it is possible to determine the pulse height the full 'shape' of the current transient is not obtainable, these events have been catagorised as 'unclassified'. Figure $3 \mathrm{~b}$ ) shows a histogram of the current heights of all of the pulse events recorded at $-1.9 \mathrm{~V}$ (vs MSRE) and in the presence of $10 \mathrm{mM} \mathrm{HClO}_{4}$. Of these nineteen individually recorded nanoparticle impact traces, there is no significant difference in the magnitude of the pulse heights for the different transient shapes. It should also be commented that as can be seen in Figure 3 a) only three categories for the full pulse shape were 
experimentally observed at $-1.9 \mathrm{~V}$ (vs MSRE) in the presence of $10 \mathrm{mM} \mathrm{HClO}_{4}$. However, examples of the fourth case, corresponding to the so called 'gradual-on, step-off' pulses, have been recorded under other experimental conditions.
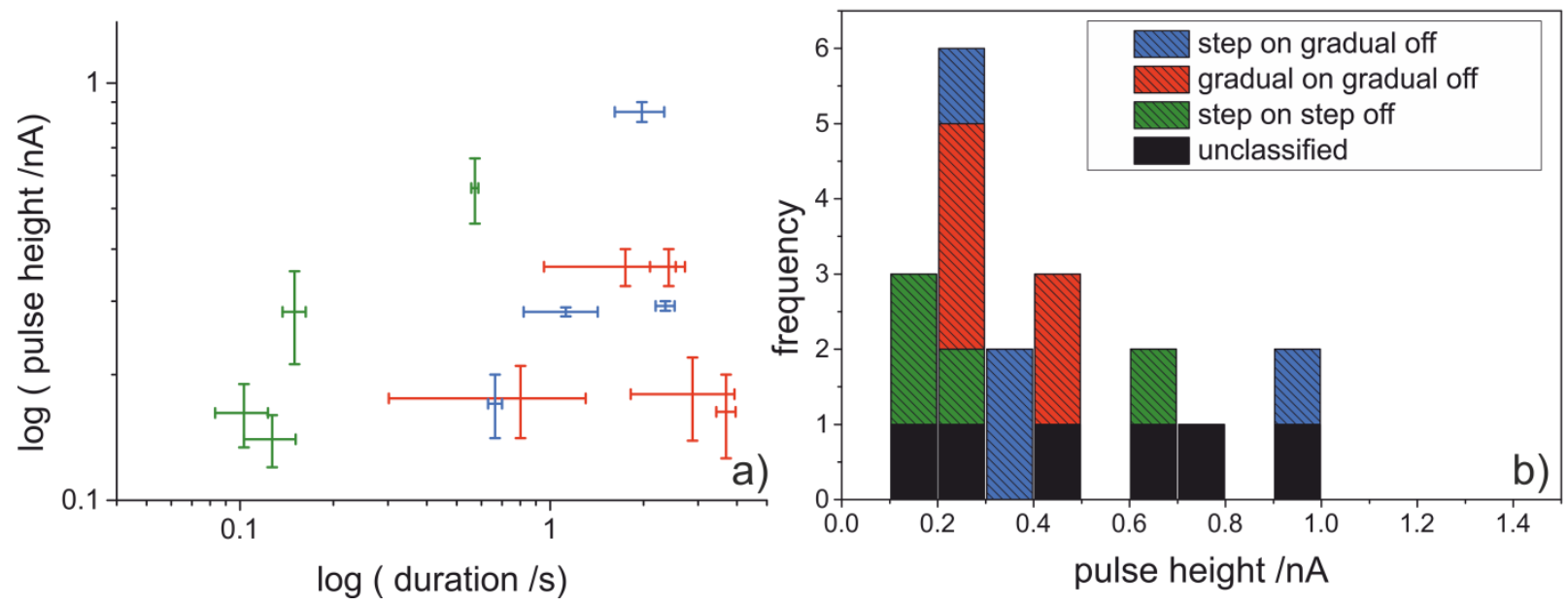

Figure 3: a) depicts a scatter plot of the pulse duration versus the pulse height (colour classifaction; blue, 'step on gradual off'; red, 'gradual on gradual off' and green, 'step on step off'. B) a histogram depicting all of the pulse heights (-1.9V [vs MRSE], $10 \mathrm{mM} \mathrm{HClO}_{4}$ as a function of the pulse shape, the unclassified category corresponds to impacts that continued beyond the experimentally recorded time frame.

With the above information three important observations can be made about these nanoparticle current traces ('pulses'). First, the impacts are stochastic in nature consequently the number of events observed per chronoamperogram is variable. Second, the residence times for the nanoparticles are relatively long $(>0.5 \mathrm{~s})$ as evidenced by the duration of the reductive pulses. This long duration implies a strong interaction between the particle and the electrode surface. Moreover, there is no indication of a correlation between the pulse duration and height. Third, as discussed above (and exemplified in Figure 2) of the recorded individual nanoparticle electrocatalytic impacts, there is marked variation of the current over the course of the pulse.

Having verified that the impacts correspond to the mediated proton reduction reaction at the individual nanoparticle, the article now turns to consider the magnitude of the current. The substrate 'shielded' diffusion limited current $\left(I_{\text {Lim }}\right)$ for an isolated particle on an electrode is given by; ${ }^{27-29}$

$I_{\text {Lim }}=4 \pi n F D_{H^{+}}\left[H^{+}\right] r_{N P} \ln 2 \quad$ Equation 1 
where $\mathrm{n}$ is the number of electrons transferred, $\mathrm{F}$ is the Faraday constant $\left(96485 \mathrm{C} \mathrm{mol}^{-1}\right)$ and all other variables are as given before. For a nanoparticle the diffusion limited current should be rapidly attained $\left(\frac{r_{N P}^{2}}{D_{H^{+}}} \sim 10^{-8} s\right)$ after application of the potential. From Equation 1 the predicted diffusion limited current to a single gold nanoparticle is calculated to be $0.72 \mathrm{nA}$ (where, $\mathrm{n}=1$, $D_{H^{+}}=8.8 \times 10^{-9} \mathrm{~m}^{2} \mathrm{~s}^{-1},\left[H^{+}\right]=10 \mathrm{~mol} \mathrm{~m}^{-3}$ and $\mathrm{r}_{\mathrm{NP}}=9.6 \mathrm{~nm}$ ). Even at $-1.9 \mathrm{~V}$ (vs MSRE) the average pulse height is significantly below this diffusion limited value. Consequently, at this potential the proton reduction is taken to be kinetically limited by the rate of electron transfer. The next section investigates the potential dependence of the nanoparticle mediated proton reduction reaction.

\section{The kinetics of proton reduction at individual gold nanoparticles}

The magnitude of the pulse heights were studied as a function of the applied electrode potential, the results of which are presented in Figure 4. As can be seen the average pulse height exhibits a significant potential dependence. Accurate determination of the pulse height below potentials of $-1.825 \mathrm{~V}$ (vs MSRE) was not feasible due to the low signal-to-noise ratio. Also as previously noted for electrode potentials greater than $-1.9 \mathrm{~V}$ (vs MSRE) the direct reduction of the protons at the carbon surface becomes appreciable. These experimental constraints lead to the relatively narrow potential window over which the catalytic impact process may be unambiguously studied.

The predicted potential dependent steady-state response for a spherical particle on a surface may be readily simulated to a high degree of accuracy by simply using the results obtained for a spherical and isolated particle. ${ }^{\mathrm{b}}$ Simulations for the steady-state response of an isolated spherical nanoparticle were undertaken using DigiSim ${ }^{\circledR}$. Through fitting of the mean pulse heights as a function of potential the apparent electrochemical rate constants for the proton reduction at an impacting gold nanoparticle can be obtained. For the impact experiments the transfer coefficient,

\footnotetext{
${ }^{\mathrm{b}}$ In order to map the two responses the rate constant and calculated current must be scaled by a factor of $\ln 2\left(I_{\text {lim,surf }}=I_{\text {lim,soln }} x \ln 2, k_{0, \text { surf }}=k_{0, \text { soln }} / \ln 2\right) .{ }^{27}$
} 
in accord with the nanoparticle ensemble response, is fixed as 0.5 . Using this model a value of $1.6 \times 10^{-9} \mathrm{~m} \mathrm{~s}^{-1}$ for the $\mathrm{k}_{0}$ is found. For comparison, the simulated responses using the kinetic data presented in Table 1 and for the best fit of the individual impacts are also depicted in Figure 4.

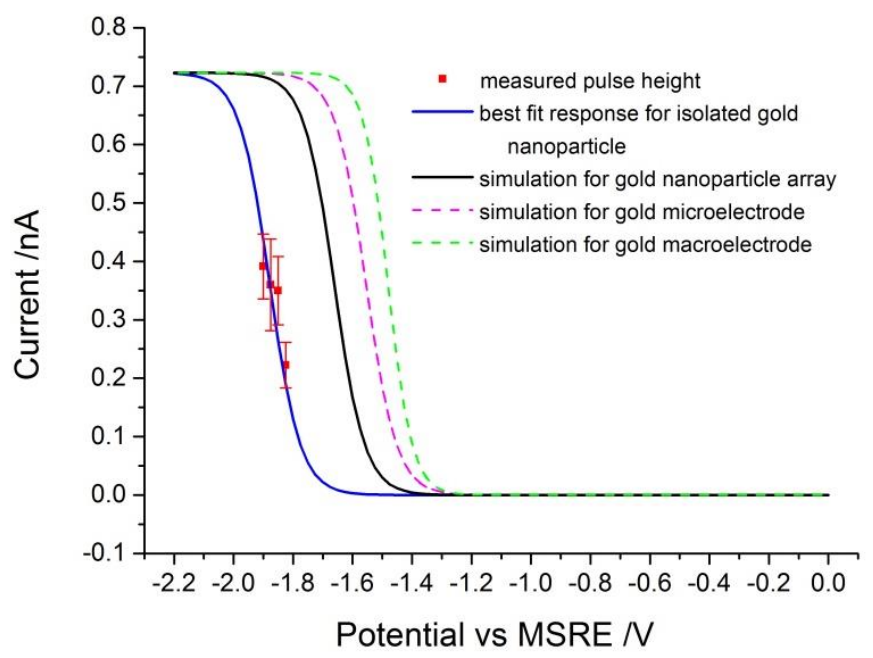

Figure 4 The measured average pulse height (red dots) as a function of the applied electrode potential. Overlaid is the simulated best fit for the experimental data (blue, $\mathrm{k0}=1.6 \times 10-9 \mathrm{~m} \mathrm{~s}-1, \alpha=0.5$ ). Also shown for comparison are the predicted responses for an isolated nanoparticle based on the kinetic parameters extracted from the gold nanoparticle array (black, k0=6.6x10-8 $\mathrm{m} \mathrm{s}-1, \alpha=0.47$ ), the gold microelectrode (pink dashed, $\mathrm{k} 0=5.3 \times 10-7 \mathrm{~m} \mathrm{~s}-1, \alpha=0.50$ ) and the gold macroelectrode (green dashed, $\mathrm{k} 0=7.1 \times 10-8 \mathrm{~m} \mathrm{~s}-1, \alpha=0.63$ ). At an applied potential of $-1.9 \mathrm{~V}$ nineteen data points were analyzed, at $-1.875 \mathrm{~V}$ ten data points were analyzed, at $-1.85 \mathrm{~V}$ seven data points were analysed and at $-1.825 \mathrm{~V}$ nine data points were analysed.

Importantly, this apparent electrochemical rate constant measured for the individual particle responses (ca. $1.6 \times 10^{-9} \mathrm{~m} \mathrm{~s}^{-1}$ ) is over an order of magnitude below that found for the average result obtained from measurements using a gold nanoparticle ensemble $\left(6.6 \times 10^{-8} \mathrm{~m} \mathrm{~s}^{-1}\right)$ : The current associated with an individual particle when in an array versus as an isolated particle differs hugely. For the present experimental case the current at an individual particle in the array at the peak current is of the order of $0.66 \mathrm{pA}$ per nanoparticle as compared to the recorded average for the impacting particles at $-1.9 \mathrm{~V}$ (vs MSRE) of $0.39 \pm 0.05 \mathrm{nA}$. This disparity in the magnitudes of the currents at the nanoparticles in the two environments reflects the altered proton diffusion fields surrounding the particles. Consequently, it is feasible that the observed change in the kinetics may relate to an interaction between the diffusion and double layers. ${ }^{7}$ 
However, such an explanation being the sole cause is unsatisfactory in its inability to account for the different impact pulse shapes.

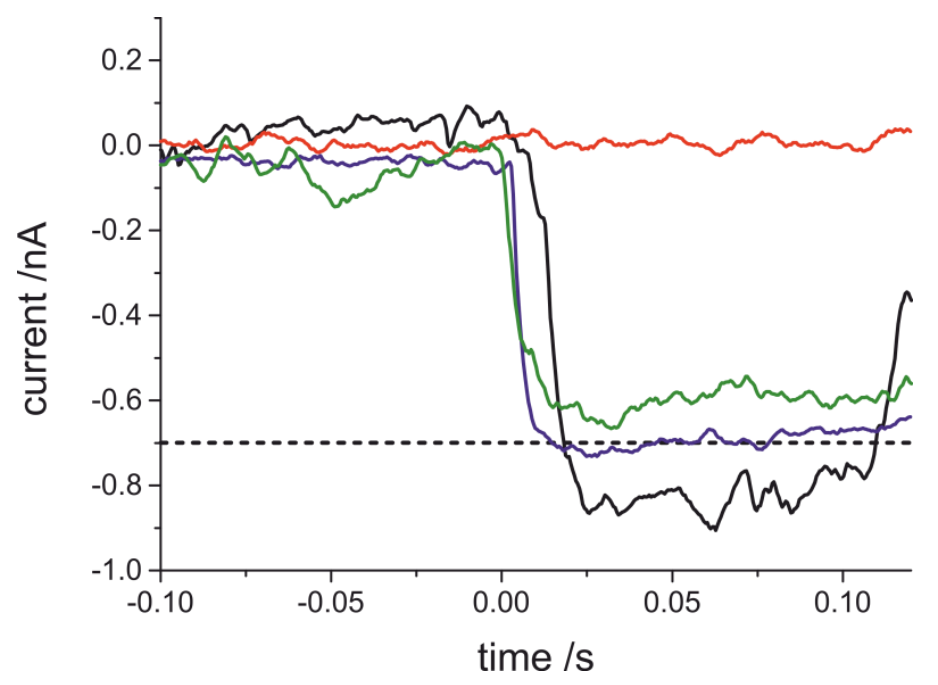

Figure 5: Example of pulse onsets for three events which exhibit near diffusion limited current (dotted horizontal line, $0.72 \mathrm{nA}$ ), red trace is an example of the 'blank' background current in the absence of a nanoparticle. Impacts recorded at $-1.9 \mathrm{~V}$ (vs MSRE) and in a $0.6 \mathrm{M} \mathrm{NaClO}_{4}, 10 \mathrm{mM} \mathrm{HClO}_{4}$ solution. Note all traces have been background corrected to allow ease of comparison.

At this stage two important interim observations can be made. First, during the course of the above experiments although the average impact current is below the expected diffusional limit some impact pulses with currents consistent with a full steady-state diffusional response at a single particle were observed. Figure 5 depicts examples of three onsets of pulse events which exhibit currents that are consistent with approximately diffusion limited currents at the impacting particles. Second, if relatively high nanoparticle concentrations are used $(60 \mathrm{pM})$ the background signal increases, reflecting the presence of multiple nanoparticles situated at the electrode surface. However, during the course of a single scan in the presence of high nanoparticle concentrations, a number of pulses are observed with a magnitude that is consistent with representing a fully diffusional current, SI section 3, as predicted by equation 1 . This result is consistent with the previously reported results for proton reduction at individual nanaoparticles. $3,30 \mathrm{c}$ These two observations seemingly imply that the activity of the electrode is

c The previous work studying gold nanoparticle impacts utilised an electrode that was electrochemically oxidatively cleaned prior to experimentation, so as to ensure the surface was 
non-uniform, such that, the probability of impacting an 'active' area of the electrode is increased with the use of higher nanoparticle concentrations. ${ }^{31}$ The final section of this article looks at the noise spectrum of the nanoparticle impacts in the presence of a higher acid concentration (20 $\mathrm{mM} \mathrm{HClO}_{4}$ ), allowing further physical insight into the interfacial processes.

\section{The Faradaic noise associated with individual nanoparticle electrocatalysis}

Figure 6 a) depicts a recorded impact response for the gold nanoparticles $(2 \mathrm{pM})$ in the presence of $20 \mathrm{mM} \mathrm{HClO}_{4}$.
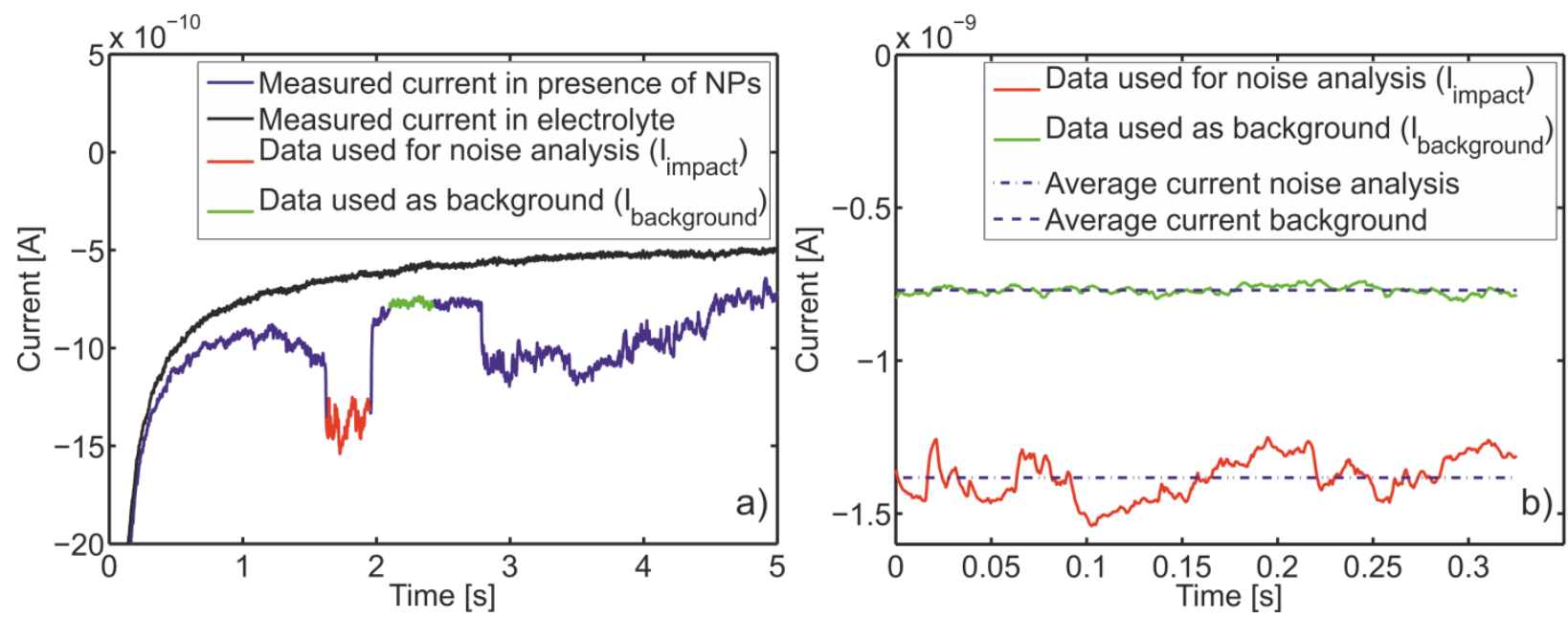

Figure 6: a) Chronoamperometric response of a carbon microelectrode potentiostated at $-1.9 \mathrm{~V}$ (vs MSRE) in a solution containing 0.6 $\mathrm{M} \mathrm{NaClO}_{4}, 20 \mathrm{mM} \mathrm{HClO}_{4}$ and $2 \mathrm{pM}$ gold nanoparticles (black trace is in the absence of nanoparticles). b) Noise fluctuations for the two highlighted regions, in the absence (green) and presence (red) of a nanoaprticle

During the course of the five second recording two clearly distinct impact pulses are detected; more importantly, during the pulses the noise spectrum is clearly altered. Figure $6 \mathrm{~b}$ ) depicts the a close-up of the two time regions as marked in Figure 6 a), the red graph presents the noise recorded during the impact and the green graph is the noise that is taken as a reference. As can be seen in the plot, the magnitude of the impact current noise in the presence of the nanoparticle is significantly larger than the background noise. One contribution to this increase in current

highly-activated. Due to the large background currents associated with such methodologies, this was note appropriate for the present work. 
fluctuations will be the shot noise associated with the Faradaic interaction of the nanoparticle with its surrounding environment. Since the average distance between two protons in solution as well as their average Brownian displacement per sampling interval do not significantly exceed the nanoparticle dimensions, only few protons react during each sampling interval. Hence, the rate of reacting protons is affected by random concentration inhomogeneities and the current fluctuates due to the discrete nature of the transferred charge per proton. These current fluctuations can be described by the shot noise model and the expected variance of the current fluctuations can be calculated as follows:

$\operatorname{Var}\left(I_{\text {Lim }}\right)=2 n e^{0} I_{\text {Lim }} \Delta f$

Equation 2

where $\mathrm{n}$ is the number of elementary charges $\mathrm{e}^{0}$ transferred per reacting analyte molecule, $\mathrm{I}_{\mathrm{Lim}}$ the limiting current per nanoparticle, and $\Delta f$ the bandwidth of the respective measurement. ${ }^{32}$ Based on the experimentally found limiting current of:

$I_{\text {Lim }}=\left|\overline{I_{\text {lmpact }}}-\overline{I_{\text {background }}}\right| \quad$ Equation 3

we can calculate the theoretically expected variance of the Faradaic current to $3.92 \times 10^{-25} \mathrm{~A}^{2}$. However, the here recorded variance:

$\operatorname{Var}\left(I_{\text {Lim }}\right)=\operatorname{Var}\left(I_{\text {impact }}\right)-\operatorname{Var}\left(I_{\text {background }}\right) \quad$ Equation 4

amounts to a significantly higher value of $4.62 \times 10^{-21} \mathrm{~A}^{2}$, which exceeds the expected Faradaic shot noise by four orders of magnitude. Hence, statistical fluctuations in the number of reacting protons cannot be the dominant source of noise in the experiment.

Since our measurement allows an immediate comparison between the background noise and the noise during the impact and changes in the noise characteristics clearly coincidence with the impact of the nanoparticle, it appears unlikely that these noise characteristics are caused by an external source. We hence presume that the recorded fluctuations and variable pulse shapes are intrinsic features of the nanoimpact and originate from the particle's nano-movement near the critical height for electron tunneling. In analogy with STM studies the contact resistance between the electrode and nanoparticle is taken to exhibit an exponential distance dependency. ${ }^{15,16}$ Consequently, the change in noise characteristics can then be understood as a fingerprint of the 
particle's movement perpendicular to the electrode surface and may be a key to understanding the particle's Brownian movement during the mediated charge transfer.

\section{CONCLUSIONS}

The HER is used as an 'inner-sphere' redox reaction for the stochastic detection of individual impacting gold nanoparticles at a potentiostated carbon microelectrode. In the present case the magnitude of the catalytic current is found to be appreciably less than that predicted for a diffusion limited rate. The magnitude of the catalytic reduction rate is experimentally shown to be dependent upon the potential held upon the carbon electrode. Consequently, the altered response of the impacting nanoparticles is understood in terms of an apparent decrease in the standard electron transfer kinetics associated with a contact resistance between the electrode and the nanoparticle. The magnitude of the contact resistance will be dependent upon the distance between the electrode and nanoparticle. Consequently, the variable currents recorded during the course of an 'impacting' particle derive from the particles nanoscopic movements above the electrode interface.

\section{ACKNOWLEDGMENTS}

The research leading to these results has received partial funding from the European Research Council under the European Union's Seventh Framework Programme (FP/2007-2013)/ERC Grant Agreement no. [320403]. K.T. was supported by a Marie Curie Intra European Fellowship within the 7th European Community Framework Programme. 


\section{REFERENCES}

(1) Dasari, R.; Robinson, D. A.; Stevenson, K. J. J. Am. Chem. Soc. 2013, 135, 570-573.

(2) Xiao, X.; Bard, A. J. J. Am. Chem. Soc. 2007, 129, 9610-9612.

(3) Kahk, J. M.; Rees, N. V.; Pillay, J.; Tshikhudo, R.; Vilakazi, S.; Compton, R. G. Nano Today 2012, 7, 174-179.

(4) Rees, N. V.; Klymenko, O. V.; Compton, R. G.; Oyama, M. J. Electroanal. Chem. 2002, 531, 33-42.

(5) Wustholz, K. L.; Henry, A.-I.; McMahon, J. M.; Freeman, R. G.; Valley, N.; Piotti, M. E.;

Natan, M. J.; Schatz, G. C.; Duyne, R. P. V. J. Am. Chem. Soc. 2010, 132, 10903-10910.

(6) Henstridge, M. C.; Ward, K. R.; Compton, R. G. J. Electroanal. Chem. 2014, 712, 14-18.

(7) Dickinson, E. J. F.; Compton, R. G. J. Electroanal. Chem. 2011, 661, 198-212.

(8) Goldman, A. J.; Cox, R. G.; Brenner, H. Chem. Eng. Sci. 1967, 22, 637-651.

(9) Bevan, M. A.; Prieve, D. C. J. Chem. Phys. 2000, 113, 1228-1236.

(10) Kazoe, Y.; Yoda, M. Appl. Phys. Lett. 2011, 99, 124104.

(11) Bard, A. J. J. Am. Chem. Soc. 2010, 132, 7559-7567.

(12) Greeley, J.; Jaramillo, T. F.; Bonde, J.; Chorkendorff, I.; Nørskov, J. K. Nat. Mater. 2006, 5, 909-913.

(13) Ogden, J. M.; Steinbugler, M. M.; Kreutz, T. G. J. Power Sources 1999, 79, 143-168.

(14) Perez, J.; Gonzalez, E. R.; Villullas, H. M. J. Phys. Chem. B 1998, 102, 10931-10935.

(15) Simmons, J. G. J. Appl. Phys. 1963, 34, 1793-1803.

(16) Hugelmann, M.; Schindler, W. Surface Science 2003, 541, L643-L648.

(17) Costentin, C.; Canales, J. C.; Haddou, B.; Savéant, J. M. J. Am. Chem. Soc. 2013, 135, 17671-17674.

(18) Zhou, J.; Zu, Y.; Bard, A. J. J. Electroanal. Chem. 2000, 491, 22-29.

(19) Brug, G. J.; Sluyters-Rehbach, M.; Sluyters, J. H.; Hamelin, A. J. Electroanal. Chem. 1984, $181,245-266$.

(20) Pentland, N.; Bockris, J. O. M.; Sheldon, E. J. Electrochem. Soc. 1957, 104, 182-194.

(21) Belding, S. R.; Campbell, F. W.; Dickinson, E. J. F.; Compton, R. G. Phys. Chem. Chem.

Phys. 2010, 12, 11208-11221.

(22) Amatore, C.; Savéant, J. M.; Tessier, D. J. Electroanal. Chem. 1983, 147, 39-51.

(23) Ward, K. R.; Gara, M.; Lawrence, N. S.; Hartshorne, R. S.; Compton, R. G. J. Electroanal. Chem. 2013, 695, 1-9.

(24) Daniel, M. C.; Astruc, D. Chem. Rev. 2004, 104, 293-346.

(25) Hernández, J.; Solla-Gullón, J.; Herrero, E.; Feliu, J. M.; Aldaz, A. J. Nanosci. Nanotech. 2009, 9, 2256-2273.

(26) Fischer, L. M.; Tenje, M.; Heiskanen, A. R.; Masuda, N.; Castillo, J.; Bentien, A.; Émneus, J.; Jakobsen, M. H.; Boisen, A. Microelec. Eng. 2009, 86, 1282-1285.

(27) Molina, A.; Gonzalez, J.; Barnes, E. O.; Compton, R. G. J. Phys. Chem. C 2014, 118, 346356.

(28) Delmastro, J. R.; Smith, D. E. J. Phys. Chem. 1967, 71, 2138-2149.

(29) Streeter, I.; Compton, R. G. J. Phys. Chem. C 2007, 111, 18049-18054.

(30) Kahk, J. M. Single nanoaprticle - electrode impact studies. Oxford University 2012.

(31) Kätelhön, E.; Cheng, W.; Batchelor-McAuley, C.; Tschulik, K.; Compton, R. G. ChemElectroChem 2014.

(32) Kätelhön, E.; Krause, K. J.; Wolfrum, B.; Compton, R. G. ChemPhysChem 2014. 
